\title{
Lensing function relation in Hadrons
}

\author{
Simone Rodini* \\ Dipartimento di Fisica, Università degli Studi di Pavia, I-27100 Pavia, Italy, \\ Istituto Nazionale di Fisica Nucleare, Sezione di Pavia, I-27100 Pavia, Italy \\ E-mail: simone.rodinio1@universitadipavia.it
}

\section{Barbara Pasquini}

Dipartimento di Fisica, Università degli Studi di Pavia, I-27100 Pavia, Italy, Istituto Nazionale di Fisica Nucleare, Sezione di Pavia, I-27100 Pavia, Italy

E-mail: barbara.pasquini@unipv.it

\begin{abstract}
Alessandro Bacchetta
Dipartimento di Fisica, Università degli Studi di Pavia, I-27100 Pavia, Italy, Istituto Nazionale di Fisica Nucleare, Sezione di Pavia, I-27100 Pavia, Italy

E-mail: alessandro.bacchetta@unipv.it
\end{abstract}

We discuss the origin of nontrivial relations between transverse distortions in the distribution of quarks in impact-parameter space and analogous distortions in transverse-momentum space due T-odd effects. We show that these relations can be established under very specific conditions, typically realized only in models that describe hadrons as two-body bound systems and involving a helicity-conserving coupling between the gauge boson and the spectator system.

XXVII International Workshop on Deep-Inelastic Scattering and Related Subjects - DIS2019

8-12 April, 2019

Torino, Italy

* Speaker. 


\section{Introduction}

Transverse distortions in the distribution of quarks in impact parameter space are described by impact-parameter dependent parton distributions (IPDs), obtained by Fourier transforms of generalized parton distributions (GPDs) that contribute to observable asymmetries in exclusive processes involving hadrons. Analogous distortion in transverse momentum space are described by transverse-momentum dependent parton distributions (TMDs) that give rise to observable asymmetries in semi-inclusive deep inelastic (SIDIS) processes. At leading twist, the correlator of IPDs has formally the same structure as the correlator for TMDs, with the impact parameter $\boldsymbol{b}_{\perp}$ taking the role of the transverse momentum $\boldsymbol{k}_{\perp}$. Beyond this formal connection, in general it is not possible to establish model-independent relations between GPDs and TMDs. Only model calculations show nontrivial relations [1-3]. The most prominent cases are the relations which describe T-odd effects in single spin asymmetries (SSAs) via factorization of the effects of final state interactions (FSIs), incorporated in a so-called "chromodynamics lensing function", and a spatial distortion of GPDs in impact parameter space [4-6]. In this contribution, we summarize the very stringent conditions for the validity of the lensing relation, that have been discussed more extensively in Ref. [7].

\section{Relations between GPDs and T-odd TMDs}

The quark TMD correlator is defined as

$$
\Phi^{[\Gamma]}\left(x, \boldsymbol{k}_{\perp}, S\right)=\left.\frac{1}{2} \int \frac{d z^{-} d z_{\perp}}{(2 \pi)^{3}} e^{i k \cdot z}\left\langle p, S\left|\bar{\psi}\left(-\frac{z}{2}\right) \Gamma \mathscr{W}\left(-\frac{z}{2}, \frac{z}{2}\right) \psi\left(\frac{z}{2}\right)\right| p, S\right\rangle\right|_{z^{+}=0},
$$

where $p=\left(p^{+}, p^{-}, \boldsymbol{p}_{\perp}=\mathbf{0}_{\perp}\right)$ and $S$ are, respectively, the hadron-target momentum ${ }^{1}$ and spin, $\psi$ is the quark field operator and $\Gamma$ is a generic matrix in the Dirac space. The TMD correlator depends on the light-cone momentum fraction $x=\frac{k^{+}}{p^{+}}$, and on the quark transverse momentum $\boldsymbol{k}_{\perp}$. The Wilson line $\mathscr{W}$ connecting the two quark fields ensures color gauge invariance and is defined as

$$
\mathscr{W}(a, b)=\mathscr{P} \exp \left\{-i g_{s} \int_{\gamma} d \zeta \cdot A(\zeta)\right\}
$$

where $g_{s}=\sqrt{4 \pi \alpha_{s}}$ and $\gamma$ is a path from $a$ to $b$ that is determined by the physical process under consideration (we consider the Wilson line of a SIDIS process). The Wilson line in Eq. (1.1) breaks the naive time-reversal invariance of the correlator and, as a consequence, T-odd TMDs need to be included. At leading-twist and for spin $1 / 2$ targets, one has two T-odd TMDs: the Sivers function $f_{1 T}^{\perp}\left(x, \boldsymbol{k}_{\perp}^{2}\right)$ and the Boer-Mulders TMD $h_{1}^{\perp}\left(x, \boldsymbol{k}_{\perp}^{2}\right)$. The Sivers function describes the momentum distribution of unpolarized quark in a transversely polarized target, and is obtained from the correlator (1.1) with $\Gamma=\gamma^{+}$. The Boer-Mulders function gives the momentum distribution of transversely polarized quarks in an unpolarized target, and is defined from the correlator (1.1) with $\Gamma=i \sigma^{j+} \gamma_{5}$. For spin- 0 targets, only the contribution of the Boer-Mulders functions can exist.

The GPDs in the momentum space are defined through the following light-cone correlation function

$$
F^{[\Gamma]}(x, \xi, t, S)=\left.\frac{1}{2} \int \frac{d z^{-}}{2 \pi} e^{i k \cdot z}\left\langle p^{\prime}, S\left|\bar{\psi}\left(-\frac{z}{2}\right) \Gamma \mathscr{W}\left(-\frac{z}{2}, \frac{z}{2}\right) \psi\left(\frac{z}{2}\right)\right| p, S\right\rangle\right|_{z^{+}=0, z_{\perp}=\mathbf{0}_{\perp}}
$$

\footnotetext{
${ }^{1}$ We use light-front coordinates, with $v^{ \pm}=1 / \sqrt{2}\left(v^{0} \pm v^{3}\right)$ and $\boldsymbol{v}_{\perp}=\left(v^{1}, v^{2}\right)$ for a generic four-vector $v$.
} 
and depend, besides $x$, on the variables $\xi=-\frac{\Delta^{+}}{2 P^{+}}$and $t=\Delta^{2}$, where $P=\left(p+p^{\prime}\right) / 2$ and $\Delta=p^{\prime}-p$.

The IPD correlator can be obtained by Fourier transform of the the GPD correlator (1.2) at $\xi=0$ from the momentum coordinates $\boldsymbol{\Delta}_{\perp}$ to the impact parameter coordinates $\boldsymbol{b}_{\perp}$, and reads

$$
\mathscr{F}^{[\Gamma]}\left(x, \boldsymbol{b}_{\perp}, S\right)=\frac{1}{2} \int \frac{d z^{-}}{2 \pi} e^{i x p^{+} z^{-}}\left\langle p^{+}, \boldsymbol{R}_{\perp}=\mathbf{0}_{\perp}, S\left|\bar{\psi}\left(z_{1}\right) \Gamma \mathscr{W}\left(z_{1}, z_{2}\right) \psi\left(z_{2}\right)\right| p^{+}, \boldsymbol{R}_{\perp}=\mathbf{0}_{\perp}, S\right\rangle,
$$

where the quark fields are evaluated at $z_{1,2}=\left(0^{+}, \mp \frac{z^{-}}{2}, \boldsymbol{b}_{\perp}\right)$ and the hadron is in a state with longitudinal momentum $p^{+}$at a transverse position $\boldsymbol{R}_{\perp}=\mathbf{0}_{\perp}$. At leading-twist and for spin $1 / 2$ targets, the correlator (1.3) with $\Gamma=\gamma^{+}$and transversely polarized targets can be parametrized in terms of the derivative of the IPD $\mathscr{E}$, while with $\Gamma=i \sigma^{j+} \gamma_{5}$ and unpolarized target we access the derivative of the combination $\mathscr{E}_{T}+2 \widetilde{\mathscr{H}}_{T}$ of chiral-odd IPDs. In the case of spin-zero targets, the contributions from the IPDs $\mathscr{E}$ and $\mathscr{E}_{T}$ are absent.

The analogy between the tensor structure of the parametrizations of the quark TMD and IPD correlators suggested a relation between T-odd TMDs and the IPDs for unpolarized (transversely) polarized quarks in a transversely polarized (unpolarized) target. In order to specify the precise form of this link, we consider the following average quark transverse momenta

$$
\left\langle k_{\perp}^{i}(x)\right\rangle_{U T}=\int d^{2} \boldsymbol{k}_{\perp} k_{\perp}^{i} \Phi^{\left[\gamma^{+}\right]}\left(x, \boldsymbol{k}_{\perp}, \boldsymbol{S}_{\perp}\right), \quad\left\langle k_{\perp}^{i}(x)\right\rangle_{T U}^{j}=\int d \boldsymbol{k}_{\perp} k_{\perp}^{i} \Phi^{\left[i \sigma^{j+} \gamma_{5}\right]}\left(x, \boldsymbol{k}_{\perp}\right),
$$

where the first and second subscripts indicate the quark and hadron polarisation, respectively. Considering the case of unpolarized quarks, we can rewrite the average transverse momentum as

$$
\begin{aligned}
& \left\langle k_{\perp}^{i}(x)\right\rangle_{U T}=\left.\frac{1}{2} \int \frac{d z^{-}}{2 \pi} e^{i x p^{+} z^{-}}\left\langle p, \boldsymbol{S}_{\perp}\left|\bar{\psi}\left(-\frac{z}{2}\right) \mathscr{W}\left(-\frac{z}{2}, \frac{z}{2}\right) \mathscr{I}^{i}\left(\frac{z}{2}\right) \gamma^{+} \psi\left(\frac{z}{2}\right)\right| p, \boldsymbol{S}_{\perp}\right\rangle\right|_{z^{+}=z_{\perp}=0} \\
& =\frac{1}{2} \int d^{2} \boldsymbol{b}_{\perp} \int \frac{d z^{-}}{2 \pi} e^{i x p^{+} z^{-}}\left\langle p^{+}, \boldsymbol{R}_{\perp}=\mathbf{0}_{\perp}, \boldsymbol{S}_{\perp}\left|\bar{\psi}\left(z_{1}\right) \mathscr{W}\left(z_{1} ; z_{2}\right) \mathscr{I}^{i}\left(z_{2}\right) \gamma^{+} \psi\left(z_{2}\right)\right| p^{+}, \boldsymbol{R}_{\perp}=\mathbf{0}_{\perp}, \boldsymbol{S}_{\perp}\right\rangle .
\end{aligned}
$$

The function $\mathscr{I}^{i}(z)$ encodes the contribution of the FSIs, and is defined as

$$
\mathscr{I}^{i}(z)=\frac{g_{s}}{2} \int d y^{-} \mathscr{W}\left(\left(z^{-}, z^{+}, z_{\perp}\right),\left(y^{-}, z^{+}, z_{\perp}\right)\right) G^{+i}\left(y^{-}, z^{+}, z_{\perp}\right) \mathscr{W}\left(\left(y^{-}, z^{+}, z_{\perp}\right),\left(z^{-}, z^{+}, z_{\perp}\right)\right)
$$

with $G^{+i}$ being the gluon-field strength tensor. In light-cone gauge $A^{+}=0$ with advanced boundary condition $\boldsymbol{A}_{\perp}\left(-\infty^{-}\right)=0$, one has $\mathscr{I}^{i}(z)=\frac{g_{s}}{2} A_{\perp}^{i}\left(\infty^{-}, z^{+}, \boldsymbol{z}_{\perp}\right)$, and Eq. (1.5) becomes

$$
\left\langle k_{\perp}^{i}(x)\right\rangle_{U T}=\left.\frac{g_{s}}{2} \int \frac{d z^{-}}{2 \pi} e^{i x p^{+} z^{-}}\left\langle p, \boldsymbol{S}_{\perp}\left|\bar{\psi}\left(-\frac{z}{2}\right) A_{\perp}^{i}\left(\infty^{-}\right) \gamma^{+} \psi\left(\frac{z}{2}\right)\right| p, \boldsymbol{S}_{\perp}\right\rangle\right|_{z^{+}=z_{\perp}=0} .
$$

One notices from Eq, (1.7) that the FSIs in the light-cone gauge with advanced boundary conditions (and, similarly, with the retarded or principal value prescriptions) reduce to the exchange of a transverse gluon at light-cone infinity between the active quark and the spectator partons. 
Using the completeness relation, we can rewrite the first line of Eq. (1.5) as

$$
\begin{aligned}
& \left\langle k_{\perp}^{i}(x)\right\rangle_{U T}=\frac{1}{2} \int\left\{d k_{1}\right\}\left\{d k_{2}\right\}\{d l\} \int \frac{d z^{-}}{2 \pi} e^{i x p^{+} z^{-}} e^{-i \frac{z^{-}}{2}\left(k_{1}^{+}+k_{2}^{+}+l^{+}\right)} \sum_{n, m} \sum_{\beta \beta^{\prime}} \int \prod_{i=1}^{n} \frac{d q_{i}^{+} d \boldsymbol{q}_{\perp, i}}{(2 \pi)^{3} 2 q_{i}^{+}} \\
& \times \prod_{j=1}^{m} \frac{d w_{j}^{+} d \boldsymbol{w}_{\perp, j}}{(2 \pi)^{3} 2 w_{i}^{+}}\left\langle p^{+}, \boldsymbol{p}_{\perp}=\mathbf{0}_{\perp}, \boldsymbol{S}_{\perp}\left|\phi\left(k_{1}\right) \gamma^{+}\right|\left\{q_{i}^{+}, \boldsymbol{q}_{\perp, i}\right\}_{n}, \beta^{\prime}\right\rangle\left\langle\left\{q_{i}^{+}, \boldsymbol{q}_{\perp, i}\right\}_{n}, \beta^{\prime}\left|I^{i}(l)\right|\left\{w_{i}^{+}, \boldsymbol{w}_{\perp, i}\right\}_{m}, \beta\right\rangle \\
& \times\left\langle\left\{w_{i}^{+}, \boldsymbol{w}_{\perp, i}\right\}_{m}, \beta\left|\psi\left(k_{2}\right)\right| p^{+}, \boldsymbol{p}_{\perp}=\mathbf{0}_{\perp}, \boldsymbol{S}_{\perp}\right\rangle,
\end{aligned}
$$

where $\{\ldots\}$ indicates the Lorentz invariant integration measure and $\phi\left(\frac{z}{2}\right)=\bar{\psi}\left(-\frac{z}{2}\right) \mathscr{W}\left(-\frac{z}{2} ; \frac{z}{2}\right)$. In Eq. (1.8), the index $\beta$ and $\beta^{\prime}$ label the parton, color and the helicity content of the intermediate states. The matrix elements of the lensing operator $I^{i}(l)$ in Eq. (1.9) represent the interaction between the active parton and the spectator system mediated by the Wilson gluons and correspond to the FSIs that occur in a SIDIS process. One can factorize the lensing function and the IPD in Eq. (1.8) by requiring that the matrix elements of the operator $I^{i}(l)$ satisfy the following relation

$$
\begin{aligned}
& \left\langle\left\{q_{i}^{+}, \boldsymbol{q}_{\perp, i}\right\}_{n}, \beta^{\prime}\left|I^{i}(l)\right|\left\{w_{i}^{+}, \boldsymbol{w}_{\perp, i}\right\}_{m}, \beta\right\rangle \\
& =2 \pi L^{i}\left(\frac{\boldsymbol{l}_{\perp}}{1-x}\right) \delta_{n, m} \delta_{\beta \beta^{\prime}} \boldsymbol{\delta}\left(l^{+}\right) \prod_{i=1}^{n}(2 \pi)^{3} 2 q_{i}^{+} \boldsymbol{\delta}\left(q_{i}^{+}-w_{i}^{+}\right) \delta\left(\boldsymbol{q}_{\perp, i}-\boldsymbol{w}_{\perp, i}-x_{i} \frac{\boldsymbol{l}_{\perp}}{1-x}\right),
\end{aligned}
$$

where $x_{i}$ is the light-cone momentum fraction of each constituent w.r.t. the hadron target lightcone momentum, i.e. $x_{i}=w_{i}^{+} / p^{+}$, and should satisfy the relation $\sum_{i} x_{i}=1-x$. The relation (1.9) imposes strict conditions that are equivalent to requiring that:

1. the FSIs should connect Fock states with the same number of constituents and the same parton, helicity and color content;

2. the FSIs should transfer the total transverse momentum $l_{\perp} /(1-x)$ to the whole spectator system;

3. the FSIs can not transfer momentum in the light-cone direction to the spectator system;

4. the FSIs should transfer a fraction $x_{i}=w_{i}^{+} / p^{+}$of the total transverse momentum to each constituent of the spectator system.

The last condition is the most stringent. It is crucial to obtain the correct transverse light-front boost that gives the non-diagonal matrix element defining the GPDs and then the transverse distortion in impact parameter space described by IPDs. In the light-cone gauge with advanced boundary conditions, one can easily deduce that the condition 4 can be realized with a coupling between the gauge boson and the spectator system only if the latter is composed by a single constituent, i.e. the hadron target is a two-body bound system. In this case, the light-cone momentum fraction of the spectator is equal to $1-x$ and the constraint on the transverse momentum transferred by the Wilson gluon to the spectator system follows trivially from the conservation of the total momentum of the hadron target. Otherwise, the condition 4 imposes to share the transverse momentum carried by the Wilson gluon with each spectator parton in a proportion equal to the longitudinal momentum fraction $x_{i}$. This can not be realised in systems composed by more than two constituents by assuming an interaction vertex between the gauge boson and a single constituent. 
We conclude that if and only if the above conditions are fulfilled we can write

$$
\begin{aligned}
& \left\langle k_{\perp}^{i}(x)\right\rangle_{U T}=-\int d k_{\perp} k_{\perp}^{i} \frac{\varepsilon_{\perp}^{j k} k_{\perp}^{j} S_{\perp}^{k}}{M} f_{1 T}^{\perp}\left(x, k_{\perp}^{2}\right) \\
& \approx \int d b_{\perp} \mathscr{L}^{i}\left(\frac{b_{\perp}}{(1-x)}\right) \mathscr{F}^{\left[\gamma^{+}\right]}\left(x, b_{\perp}, S_{\perp}\right)=\int d b_{\perp} \mathscr{L}^{i}\left(\frac{b_{\perp}}{(1-x)}\right) \frac{\varepsilon_{\perp}^{j k} b_{\perp}^{j} S_{\perp}^{k}}{M}\left(\mathscr{E}\left(x, b_{\perp}^{2}\right)\right)^{\prime} .
\end{aligned}
$$

Analogously, we can analyze the average quark transverse momentum of a transversely polarized quark in an unpolarized target and establish a lensing relation between the Boer-Mulders function and the combination of IPDs $-\left(\mathscr{E}_{T}\left(x, \boldsymbol{b}_{\perp}^{2}\right)+2 \widetilde{\mathscr{H}}_{T}\left(x, \boldsymbol{b}_{\perp}^{2}\right)\right)^{\prime}$, with $\mathscr{E}_{T}\left(x, b_{\perp}^{2}\right)=0$ and $2 \widetilde{\mathscr{H}}_{T} \rightarrow \widetilde{\mathscr{H}}_{T}$ for spin-zero targets.

Examples of models that satisfy the relations 1-4 are the scalar-diquark spectator model $[1,6]$, or the axial-diquark models that admit only transverse polarization for the axial-vector diquark for the nucleon [8], and relativistic models for the pion at the lowest order in the Fock-space expansion $[9,10]$. Viceversa, they are violated within three-quark model calculations for the nucleon [11-15].

Using the representation of the TMDs and GPDs in terms of light-front wave functions (LFWFs), we can explicitly show how the the lensing relation is obtained in the case of the pion, and how it is violated in the case of the three-quark model calculations for the proton.

In the case of the pion, described as a quark-antiquark bound system, the GPD and the BoerMulders TMD can be written as [10]

$$
\begin{aligned}
& \frac{\Delta_{\perp}^{k}}{2 M_{\pi}} \widetilde{H}_{T, \pi}\left(x, 0,-\Delta_{\perp}^{2}\right)=\frac{T_{\pi}^{2}}{2(2 \pi)^{3}} \int d \boldsymbol{k}_{\perp} G^{k}\left(x, \boldsymbol{k}_{\perp}|| x, \boldsymbol{k}_{\perp}+(1-x) \boldsymbol{\Delta}_{\perp}\right), \\
& k_{\perp}^{k} h_{1, \pi}^{\perp}\left(x, \boldsymbol{k}_{\perp}^{2}\right)=\frac{2 \alpha_{s}}{(2 \pi)^{4}} \frac{4}{3} T_{\pi}^{2} M_{\pi} \int \frac{d \boldsymbol{q}_{\perp}}{q_{\perp}^{2}} G^{k}\left(x, \boldsymbol{k}_{\perp}|| x, \boldsymbol{k}_{\perp}-\boldsymbol{q}_{\perp}\right),
\end{aligned}
$$

where $\boldsymbol{q}_{\perp}$ is the transverse momentum of the Wilson gluon and the function $G$ is defined from the light-front wave amplitude (LFWA) overlap $F^{k}$ as [7]

$$
G^{k}\left(x_{1}, \boldsymbol{k}_{\perp, 1}|| x_{1}^{\prime}, \boldsymbol{k}_{\perp, 1}^{\prime}\right)=F^{k}\left(x_{1}, \boldsymbol{k}_{\perp, 1} ; 1-x,-\boldsymbol{k}_{\perp, 1}|| x_{1}^{\prime}, \boldsymbol{k}_{\perp, 1}^{\prime} ; x_{1}^{\prime},-\boldsymbol{k}_{\perp, 1}^{\prime}\right) .
$$

In Eq. (1.13), the arguments of the functions on the right-hand (left-hand) side of $\|$ refer to the momentum dependence of the complex conjugate LFWF of the pion in the final (initial) state. With the formal identification of

$$
-\boldsymbol{q}_{\perp}=(1-x) \boldsymbol{\Delta}_{\perp}
$$

one obtains:

$$
\left\langle k_{\perp}^{i}\right\rangle_{T U}^{j}=\int d \boldsymbol{b}_{\perp} \frac{\varepsilon_{\perp}^{k j} b_{\perp}^{k}}{M_{\pi}} \mathscr{L}_{\perp}^{i}\left(x, \boldsymbol{b}_{\perp}\right)\left(\widetilde{\mathscr{H}}_{T, \pi}\left(x, \boldsymbol{b}_{\perp}^{2}\right)\right)^{\prime},
$$

with the lensing function [6]

$$
\mathscr{L}^{i}\left(\boldsymbol{b}_{\perp} /(1-x)\right)=-i \frac{4}{3} \alpha_{s} 4 \pi \int \frac{d \boldsymbol{q}_{\perp}}{q_{\perp}^{2}} \boldsymbol{q}_{\perp}^{i} e^{-i \frac{\boldsymbol{b}_{\perp} \cdot \boldsymbol{q}_{\perp}}{(1-x)}}=-\frac{8}{3} \alpha_{s} 4 \pi^{2} \frac{b_{\perp}^{i}}{\boldsymbol{b}_{\perp}^{2}}(1-x) .
$$

Note that the relation in Eq. (1.14) is exactly the condition 4. 
For the proton, described as three-quark bound system, we introduce the appropriate LFWA overlap $G_{T}[7,11]$ and obtain the following expressions for the GPD and for the Sivers function:

$\frac{i \varepsilon_{\perp}^{i j} \Delta_{\perp}^{j} \boldsymbol{S}_{\perp}^{i}}{M} E\left(x,-\Delta_{\perp}^{2}\right)=\int \frac{d \boldsymbol{k}_{\perp}}{2(2 \pi)^{3}} \int_{0}^{x} d y \int \frac{d \boldsymbol{t}_{\perp}}{2(2 \pi)^{3}} G_{T}\left(x, \boldsymbol{k}_{\perp} ; y, \boldsymbol{t}_{\perp}|| x, \boldsymbol{k}_{\perp}+(1-x) \boldsymbol{\Delta}_{\perp} ; y, \boldsymbol{t}_{\perp}-y \boldsymbol{\Delta}_{\perp}\right)$,

$\frac{\varepsilon_{\perp}^{i j} k_{\perp}^{j} \boldsymbol{S}_{\perp}^{i}}{M} f_{1 T}^{\perp}\left(x, \boldsymbol{k}_{\perp}^{2}\right)=-\frac{\alpha_{s}}{3(2 \pi)^{7}} \int \frac{d \boldsymbol{q}_{\perp}}{q_{\perp}^{2}} \int_{0}^{x} d y \int d \boldsymbol{t}_{\perp} G_{T}\left(x, \boldsymbol{k}_{\perp} ; y, \boldsymbol{t}_{\perp}|| x, \boldsymbol{k}_{\perp}-\boldsymbol{q}_{\perp} ; y, \boldsymbol{t}_{\perp}+\boldsymbol{q}_{\perp}\right)$.

From this expression, one clearly sees that the formal identification in Eq. (1.14) does not apply, since $(1-x)$ and $y$ are independent variables. This break the condition 4 and, hence, the validity of the lensing relation.

\section{Acknowledgments}

This work is supported by the European Research Council (ERC) under the European Union's Horizon 2020 research and innovation programme (grant agreement No. 647981, 3DSPIN).

\section{References}

[1] S. Meissner, A. Metz and K. Goeke, Phys. Rev. D 76 (2007) 034002.

[2] H. Avakian, A. V. Efremov, P. Schweitzer and F. Yuan, Phys. Rev. D 81 (2010) 074035.

[3] C. Lorcé and B. Pasquini, Phys. Rev. D 84 (2011) 034039.

[4] M. Burkardt, Phys. Rev. D 69 (2004) 057501.

[5] M. Burkardt, Nucl. Phys. A 735 (2004) 185.

[6] M. Burkardt and D. S. Hwang, Phys. Rev. D 69 (2004) 074032.

[7] B. Pasquini, S. Rodini and A. Bacchetta, arXiv:1907.06960 [hep-ph]

[8] A. Bacchetta, F. Conti and M. Radici, Phys. Rev. D 78 (2008) 074010.

[9] L. Gamberg and M. Schlegel, Phys. Lett. B 685 (2010) 95.

[10] B. Pasquini and P. Schweitzer, Phys. Rev. D 90 (2014) no.1, 014050.

[11] B. Pasquini and F. Yuan, Phys. Rev. D 81 (2010) 114013.

[12] B. Pasquini, S. Cazzaniga and S. Boffi, Phys. Rev. D 78 (2008) 034025.

[13] S. Boffi, B. Pasquini and M. Traini, Nucl. Phys. B 649 (2003) 243.

[14] B. Pasquini, M. Pincetti and S. Boffi, Phys. Rev. D 72 (2005) 094029.

[15] B. Pasquini and S. Boffi, Phys. Lett. B 653 (2007) 23. 